\title{
Deformation Measurements of Ballast-less Railway Line
}

Jana Ižvoltová*, Tomáš Cesnek

Department of Geodesy, University of Žilina, Univerzitná 8215/1, 01026 Žilina

\begin{abstract}
New technologies of absolute and relative measurements of a railway line based on continual observation techniques bring a new approach to data processing. The paper is devoted to the process of data manipulation, evaluation and analysis by using methods of numerical mathematics with aim to obtain precise, reliable and objective results, which define the track geometry in conformity with the appropriate technical standards and instructions.
\end{abstract}

\section{Introduction}

Present monitoring the railway line involves absolute and relative techniques, which are based on discrete and continual technologies of data acquisition. While the discrete measurements belong to the conventional methods, the modern one exchange manual measuring devices for automatized measuring vehicles with the continual data recording. However, the observation process has become more time efficient, the evaluation procedures have changed due to clouds of data recording. Besides, the new measuring technologies bring also changes into appropriate technical standards, which modify their requirements on diagnostics of a railway line. Specifically, the Slovak Technical Standard STN 736360 [1] primary recommends the continual measurements of track geometry, though the discrete observations are accepted in rare cases. The complex diagnostics of the

\footnotetext{
${ }^{*}$ Corresponding author: jana.izvoltova@fstav.uniza.sk
} 
actual railway condition anticipate the combination both of relative and absolute observation techniques, which lead to determining the relative track parameters as track gauge, cross-level and track gradient and absolute track characteristics represented by the longitudinal, directional and height track position. defined in the global coordination and height systems. The paper is devoted to the process of evaluation of clouds of data acquired by the absolute geodetic techniques, which involves the numerical solutions need to control the stability of benchmarks, to construct the mathematical model of railway line by the regression method and to qualify the actual track condition by the method of deformation analysis.

\section{Methods of Railway Line Diagnostics}

Absolute conventional methods of railway line utilized geodetic discrete measurements of polar coordinates (angle, distance). Three basic methods used to be utilized in the railway line measurements, in the past:

- Method of arc coordinates, which utilized mathematical relations between arc chord, chamber and radius.

- Polygonal method based on measurement of polar coordinates in polygon stabilized right in the railway line axes.

- Long chord method based on the relationship between height of arc and it's long chord.

In case of deformation analysis of track geometry, the evaluation of discrete measurements process was based on mutual comparison of the appropriate discrete points obtained by the periodical measurements. So, the observations process assumed to minimize a pointing error by adding precise signalling devices fixed at the rail body (see fig 1.) or placed on the gauge bar right in the track axis.
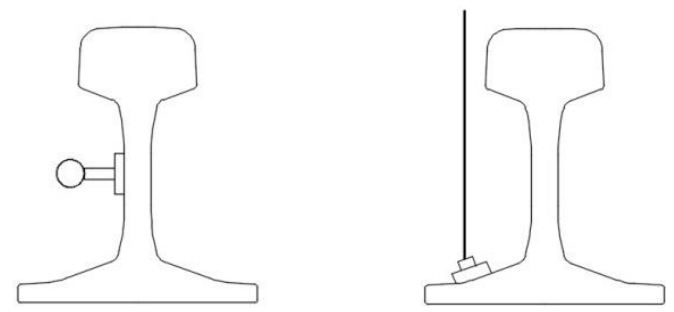

Fig. 1 An example of additive devices for discrete measurement of track geometry 
The present relative track diagnostics is realized by a gauge vehicle to measure the main relative track characteristics as gauge, vertical and horizontal alignment, cant and twist. Sophisticated software recalculates measured data to real track geometry, and displays measurement history, events, local defects and quality indices. The actual absolute track diagnostics represent precise levelling, electronical surveying, 3D laser scanning, satellite observations, etc.. The advanced track diagnostics technology is based on multisensory, which combine both of relative and absolute techniques into automated track vehicle for continual recording and surveying the railway body and it's environment. Such a track "trolley" is characterized by an amount of recording data, which require a special hardware and software to manipulate and evaluate with them by using methods of numerical mathematics. The actual diagnostics method of track geometry is realized in conformity with the appropriate technical standards [4], [5] and [6].

\section{Numerical Analysis of Reference System}

Geodetic measurements of railway line are connected to the National Spatial Network via railway benchmarks formed into spatial reference system. The positional and height stability of the reference system belongs to the first requisite to ensure the reliable results. Numerical mathematics provides several methods to control the stability of the spatial reference system. Very useful seems to be seven parameters similarity transformation method, which can be expressed as:

$$
\mathbf{X}^{\prime}=q \cdot \mathbf{R} \mathbf{X}+\mathbf{X}_{\mathbf{0}},
$$

where $\mathbf{X}^{\prime}$ is the transformed vector, $\mathbf{X}$ is the initial vector, $\mathbf{X}_{\mathbf{0}}$ is translation vector, $\mathbf{R}$ is rotation matrix and $q$ is a scale factor. An application of this method requires an adequate number of identical points. In case of redundancy the least square method is recommended to find an unbiased estimation. The estimated vector of residuals give the view on a possible spatial discrepancy of a benchmark, which should be analysed by a method of outlier diagnostics.

Marcak and Kubacek published in [2] sophisticated method used to control vertical reference network by the method of regression analysis. The method involves parameter estimation of a regression line constructed from a cumulative function of elevation differences (superelevations) between all pairs of the reference points:

$$
\Delta_{i, i+1}^{j}=\sum_{k=i}^{n-1} \Delta h_{k, k+1}^{j},
$$

where the upper index $j$ is the series of measurement, lower index $i, i+1$ represents the pairs of points, in which the superelevation $\Delta$ is calculated and $n$ is the number of all reference points. The elevation $\Delta h$ represents the levelling difference between a point of 
an actual measurement and the appropriate one comes from the first measurement as follows:

$$
\Delta h_{k, k+1}^{j}=h_{k, k+1}^{j}-h_{k, k+1}^{1},
$$

Providing, the superelevations are normally distributed, the regression line can be estimated by least square method, in other case a robust regression is recommended (Cebecauer [6], Rousseeuw [7]).

The final advisement of the reference network stability is realized by the statistical hypothesis testing. A null hypothesis is proposed for the statistical relationship between an extreme value and the critical one, both from the same probability distribution:

$$
H_{0}: x_{e x}>t_{\alpha} \sigma
$$

where $t_{\alpha}$ is a quantile of Student's $t$-distribution and $\sigma$ is standard deviation, which defines an internal accuracy of the reference system.

\section{Regression analysis}

A regression model is used to substitute the real continual measurement with a mathematical function with aim to study the track directional and longitudinal parameters to predict the railway line behaviour. Developing of the mathematical function depends on the directional and height design parameters of the railway line.

In case of curve section of the railway line, the quadratic nonlinear regression model used to be applied to reconstruct the curve parameters of the railway line:

$$
R^{2}=\left(x_{i}-X_{S}\right)^{2}+\left(y_{i}-Y_{S}\right)^{2},
$$

where $\mathrm{R}$ is an unknown radius of a curve, $X_{s}, Y_{s}$ are also unknown coordinates of a curve centre and $x_{i}, y_{i}$ are known coordinates of a $i$-th measured point.

In case of spiral section of railway line, the cubic nonlinear model is developed to estimate the unknown parameters $a, b, c, d$ of cubic parabola, as follows:

$$
y_{i}=a x_{i}^{3}+b x_{i}^{2}+c x_{i}+d .
$$

The linear section of railway line can be estimated by linear function:

$$
y_{i}=a+b x_{i}
$$

where $a, b$ represents intercept and slope of line.

Evaluation of the above mentioned mathematical functions anticipates an application of a method of estimation theory to find the unknown parameters. An unbiased estimation is 
possible to achieve by the application of the collocation method, least square method, robust method, maximum like hood estimation, unbiased quadratic estimation, etc.

\section{Deformation Analysis}

The analysis of track changes belongs to the statistical analysis testing based on postulate of null hypothesis:

$$
H_{0}: \Delta Z=0
$$

where $\Delta Z$ is real track displacement, which depends on real observation error $\varepsilon_{\Delta z}$ and the measured displacement $\Delta z$

$$
\Delta Z=\Delta z+\varepsilon_{\Delta z}
$$

Suppose, the measured values are normal distributed the confidential interval for identification the measured displacement can be defined as follows:

$$
P\left(\left|\varepsilon_{\Delta z}\right|>t_{\alpha} \sigma_{\Delta z}\right)=P\left(|\Delta z|>t_{\alpha} \sigma_{\Delta z}\right)=\alpha
$$

where $\sigma_{\Delta z}$ is standard deviation depended on random variances of both of series of observations:

$$
\sigma_{\Delta z}=\sqrt{\sigma_{z 1}^{2}+\sigma_{z i}^{2}}
$$

Deformation analysis provides three possibilities to identify the track displacement [7]:

- If $|\Delta z|<\sigma_{\Delta z}$ null hypothesis is accepted and a point displacement is not evident

- If $\sigma_{\Delta z} \leq|\Delta z|<2 \sigma_{\Delta z}$ a point, displacement is regarded to be possible

- If $|\Delta z|>2 \sigma_{\Delta z}$ null hypothesis is not accepted and a point displacement is evident

\section{Mathematical Modelling}

In Slovakia, the railway modernization brings except of social-economic and environmental advantages also the technological innovations of construction layers of railway body. Such a new technology consist in replacement of the traditional railway body by the ballast-less track construction. The specialists of the Department of Geodesy and Department of Railway Construction of the University of Zilina have been asked to monitor such a new 
railway construction with aim to diagnose its behaviour in time of traffic loading. Up to time, five periodical measurements of absolute and relative track measurements were realized to define track geometry of about $2 \mathrm{~km}$ long ballast-less railway line, see fig. 1, 2 .

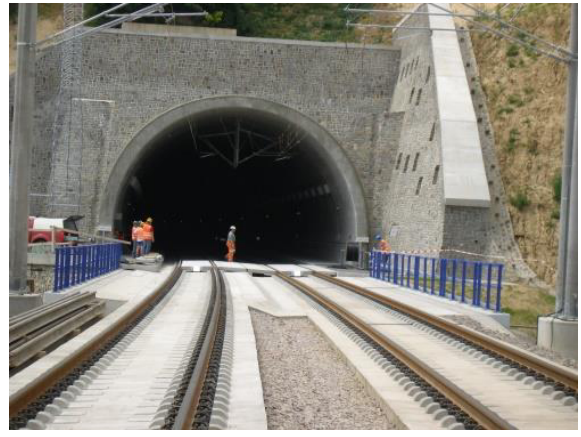

Fig. 2 Bridge part of ballast-less

track construction

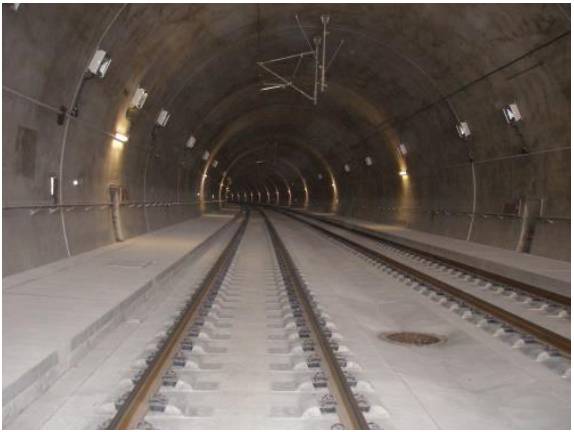

Fig. 3. Tunnel part of ballast-less track construction

Evaluation process of continual observations realized by the automatized railway vehicle starts with mathematical modelling by applying mixed Gauss-Markov model [8], [9], which deterministic formula can be written as:

$$
\mathbf{B}^{T} \mathbf{v}+\mathbf{A d} \mathbf{d}+\mathbf{u}=0
$$

where $\mathbf{A}, \mathbf{B}$ and $\mathbf{u}$ represent matrices of known parameters, $\mathbf{d x}$ is a vector of unknown values and $\mathbf{v}$ is vector of residuals. Least square method was applied to estimate unknown parameters of all railway line sections (curve, transit curve, line) and accuracy parameters:

$$
\Omega=\mathbf{v}^{T} \mathbf{P v}+2 \mathbf{k}^{T}\left(\mathbf{B}^{T} \mathbf{v}+\mathbf{A} d \mathbf{x}+\mathbf{u}\right)=\min .
$$

The solution of equation (13) leads to multiplying block matrices:

$$
\left(\begin{array}{c}
\mathbf{k} \\
-\mathbf{d x}
\end{array}\right)=\left(\begin{array}{cc}
\mathbf{B}^{T} \mathbf{Q} \mathbf{B} & \mathbf{A} \\
\mathbf{A}^{T} & 0
\end{array}\right)^{-1}\left(\begin{array}{l}
\mathbf{u} \\
0
\end{array}\right)
$$

where $\mathbf{k}$ is unknown vector of Lagrange coefficients. Accuracy parameters are represented by the covariance matrix, which is estimated according to the equation:

$$
\mathbf{D}(\mathbf{x})=\sigma^{2}\left(-\left(\mathbf{A}^{T}\left(\mathbf{B}^{T} \mathbf{Q} \mathbf{B}\right)^{-1} \mathbf{A}\right)^{-1}\right)
$$

The estimation of unit variance depends inter alia on the number of measured values $n$ and the number of unknown parameters $k$ as follows: 


$$
\sigma^{2}=\frac{\mathbf{v}^{T} \mathbf{P v}}{n-k},
$$

\section{Conclusion}

Standard deviation subjected to the precision analysis in equation (21) originates from the diagonal elements of covariance matrix in (13) and represents the a-posteriori accuracy of transversal track changes. It involves the error of multitrack target positioning in regard of track axis, which is $\pm 2 \mathrm{~mm}$ and accuracy of estimation process, which is about $\pm 2-3 \mathrm{~mm}$. As we can see in fig. 1 the transversal displacement of ballast-less track was not confirmed and the estimated values of track changes are in the frame of measuring errors.

\section{Acknowledgements}

This article is the result of the implementation of the project VEGA 1/0275/17 "Application of numerical methods to define the changes of geometrical track position " supported by the Scientific Grant Agency of the Ministry of Education, science, research and sport of the Slovak Republic and the Slovak Academy of Sciences.

This article is the result of the implementation of the project ITMS 26220220156 "Broker centre of air transport for transfer of technology and knowledge into transport and transport infrastrukture" supported by the Research \& Development Operational Programme funded by the ERDF.

\section{References}

1. STN 736360 Railway applications. Track. Part 1: Geometrical position and arrangement of $1435 \mathrm{~mm}$ gauge Railways, Slovak Office of Standards, Metrology and Testing, Bratislava (2015)

2. P Marcak, L. Kubacek. L.: Studia Geophysica et Geodaetica, 18, 33-46, 1974

3. L. Ižvolt, S. Hodás, J. Šestáková, Railway buildings 1. Design, building, construction of railway lines and stations. Handbook, EDIS (2015)

4. STN 730275 Control measurements of line buildings. Precision of geometrical parameters in construction. SÚTN (1985)

5. STN 730405 Measurement of building deformations. SÚTN (1985)

6. Direction SR 103- (S) Common demands on design, building, repair, maintenance and acceptance of work realized on ballast-less track construction. Bratislava

7. J. Gašinec, S. Gašincová, J. Sabová, G., 14. conference of Mining Surveyors and Geologists, Ostrava VŠB TU, (2007) 
8. W. Niemeier, Ausgleichungsrechnung. Walter de Gruyter GmbH \& Co. Berlin (2001)

9. A. Villim, J. Mužík, Analysis of satellite and terrestrial measurements in 3D geodetic network for transport infrastructure, Juniorstav 15, Brno (2013) 